\title{
Determinative Structural Features Identified With Probe-based Confocal Endomicroscopy for the Accurate Diagnosis of Gallbladder Malignancy
}

\author{
TOMOTAKE KOIZUMI ${ }^{1}$, TAKESHI AOKI ${ }^{1}$, TOSHIKO YAMOCHI ${ }^{2}$, YOSHIHIKO TASHIRO ${ }^{1}$, \\ TATSUYA YAMAZAKI ${ }^{1}$, TOMOKAZU KUSANO ${ }^{1}$, KAZUHIRO MATSUDA $^{1}$, YUSUKE WADA ${ }^{1}$, \\ HIDEKI SHIBATA ${ }^{1}$, KAZUHIKO SAITO ${ }^{1}$, KOJI NOGAKI ${ }^{1}$, TOMOKI HAKOZAKI ${ }^{1}$, \\ KODAI TOMIOKA ${ }^{1}$, TAKAHITO HIRAI ${ }^{1}$, AKIRA FUJIMORI ${ }^{1}$, DOAA A. MANSOUR ${ }^{1,3}$, \\ AHMED ELEWA ABBAS GAHIN ${ }^{1,4}$ and ROBERT M. HOFFMAN ${ }^{5,6}$ \\ ${ }^{1}$ Division of Gastroenterological and General Surgery, \\ Department of Surgery, School of Medicine, Showa University, Tokyo, Japan; \\ ${ }^{2}$ Department of Pathology and Laboratory Medicine, School of Medicine, Showa University, Tokyo, Japan; \\ ${ }^{3}$ General Surgery Department, Cairo University Hospitals, Cairo, Egypt; \\ ${ }^{4}$ General Surgery Department, National Hepatology and Tropical Medicine Research Institute, Cairo, Egypt; \\ ${ }^{5}$ Department of Surgery, University of California, San Diego, CA, U.S.A.; \\ ${ }^{6}$ AntiCancer Inc., San Diego, CA, U.S.A.
}

\begin{abstract}
Background: Probe-based confocal laser endomicroscopy ( $p C L E$ ) can visualize microscopic structures at high resolution but has not yet yielded definitive diagnostic features of gallbladder malignancy, as opposed to benign changes. Patients and Methods: A total of 73 patients had their gallbladder evaluated with $p C L E$ performed on resected benign and malignant gallbladder surgical specimens, which were sprayed with fluorescein. Malignant and benign features of pCLE findings were identified on the basis of Miami and Paris Classifications. Standard histopathological diagnoses and individual patient $p C L E$ findings of gallbladder lesions were correlated. Results: Of the 73 consecutive patients that had their gallbladder evaluated ex vivo with $p C L E, 11$ were identified with gallbladder malignancy. pCLE identified features of gallbladders examined ex vivo, including the presence of thick dark bands and dark clumps, which together correlated with histopathologically-determined biliary
\end{abstract}

This article is freely accessible online.

Correspondence to: Takeshi Aoki, MD, Ph.D., Division of Gastroenterological and General Surgery, Department of Surgery, School of Medicine, Showa University, 1-5-8 Hatanodai, Shinagawa-ku, Tokyo 1428666, Japan. Tel: +81 337848541, Fax: +81 337845835, e-mail: takejp@med.showa-u.ac.jp

Key Words: Gallbladder cancer, microscopy, confocal, endomicroscopy diagnosis, malignancy. malignancy at 100\% sensitivity. Thick white bands and visualized epithelium, also identified with pCLE, together correlated with histopathologically-determined malignancy at 100\% specificity. Conclusion: pCLE can be used for real-time differentiation of cancerous/non-cancerous regions in the gallbladder using the diagnostic criteria identified in the present study.

Although gallbladder cancer is the most common cancer of the biliary tract (1), it is one of the most difficult cancers to diagnose.

Probe-based confocal laser endomicroscopy (pCLE) is an imaging modality that allows in vivo microscopic imaging of the biliopancreatic tree (2-7). pCLE has the potential to acquire real-time histological information from biliary and pancreatic-duct tissues (6). pCLE findings in the pancreaticobiliary ducts were described and organized as the Miami classification (8). Subsequently, the Paris classification was proposed to refine this classification and improve the diagnostic accuracy of benign inflammatory structures (9). However, neither classification was able to agree on definite structural features visualized by pCLE that accurately determine gallbladder malignancy. The present study used resected benign and malignant gallbladder specimens, sprayed with fluorescein and imaged with pCLE, to visualize structures that accurately determined gallbladder malignancy. The present study demonstrated pCLE has clinical benefits for patients to determine if their gallbladder disease is malignant. 

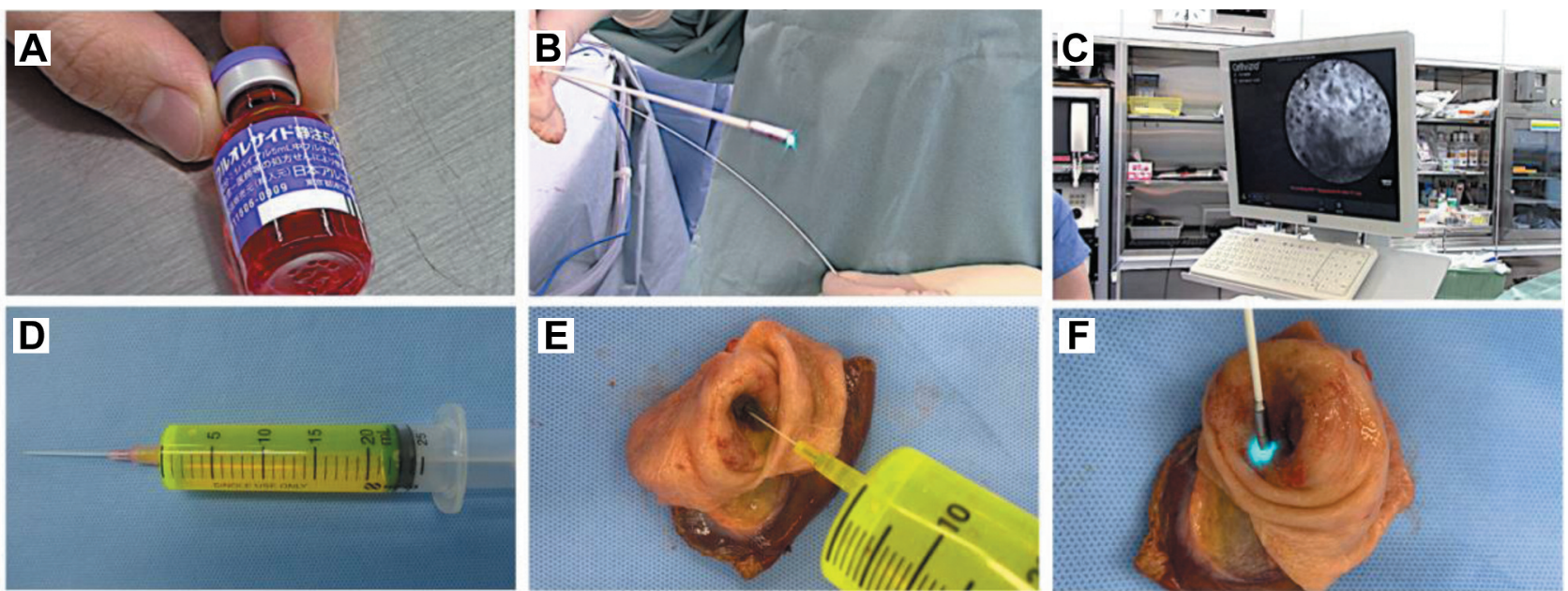

Figure 1. Ex vivo probe-based confocal laser endomicroscopy ( $p C L E)$ imaging of gallbladder cancer using resected surgical specimens. A: Source of fluorescein (10\%, Alcon Japan Co., Tokyo) used in the study. B: Gastroflex ${ }^{\circledR}$ image probe. C: Cellvizio ${ }^{\circledR}$ image. D: Diluted fluorescein solution used to spray the gallbladder specimen. E: Spraying of diluted fluorescein solution onto the surgical gallbladder specimen for tissue contrast. F: Ex vivo $p C L E$ observation of gallbladder with manual contact of $p C L E$ probe with the specimen.

\section{Patients and Methods}

Patients. This observational, single-center study utilized retrospective data collected from the Department of Surgery, Showa University, Tokyo, Japan, from April 2016 to March 2020. A total of 73 consecutive patients had ex vivo pCLE observation of their surgical gallbladder specimens.

This study was approved by the Ethics Committee of Showa University (Institutional Renew Board protocol: 1685) and was performed in accordance with the Helsinki Declaration. Written informed consent was obtained from all patients.

Ex vivo microscopic imaging using $p C L E$. Ex vivo gallbladder imaging used surgical specimens from patients who underwent surgery, including cholecystectomy, and was performed using a Cellvizio ${ }^{\circledR}$ pCLE system (Mauna Kea Technologies, Paris, France). A total of 62 cases which were histopathologically diagnosed with benign diseases after surgery were included among the 73 patients in the study. For ex vivo observation, the lumen of the gallbladder was opened following surgical resection, after which $0.1 \mathrm{ml}$ of a $10 \%$ sodium-fluorescein solution, diluted in $20 \mathrm{ml}$ normal saline, was sprayed onto the surgical specimen for tissue contrast (Figure 1) (10). pCLE observation was generally possible 1 min after spraying with fluorescein. Ex vivo microscopic examination with pCLE was performed using a Miniprobe ${ }^{\circledR}$ (Mauna Kea Technologies) or Gastroflex ${ }^{\circledR}$ (Mauna Kea Technologies) via direct manual contact of the probe tip with the luminal surface of the area to be inspected after resection. pCLE imaging was used to scan the ex vivo specimen, after which microscopic video sequences from the regions of interest, as indicated by the investigator, were acquired and saved.

The pCLE findings via ex vivo observation of each case were interpreted as either malignant or benign features based on the Miami Classification (8) and Paris Classification (9). Three
Table I. Pathological diagnosis and surgical procedures for patients in the study.

\begin{tabular}{lr}
\hline & $\mathrm{n}$ \\
\hline Diagnosis & \\
$\quad$ Adenocarcinoma & 11 \\
Intracystic papillary neoplasm & 1 \\
Cholesterol polyp & 2 \\
Chronic cholecystitis & 55 \\
Gangrenous cholecystitis & 5 \\
Adenomyomatosis & 7 \\
Surgical procedure & \\
Laparoscopic cholecystectomy & 6 \\
Extended cholecystectomy + lymphadenectomy & 17 \\
Bisegmental liver resection (S 4b/5) & 1 \\
Extrahepatic bile-duct resection + cholecystectomy & 3 \\
Partial liver resection+cholecystectomy & 8 \\
S4 hepatic subsegmentectomy & 1 \\
S5 hepatic subsegmentectomy & 1 \\
Right hepatic lobectomy & 2 \\
Left hepatic lobectomy & 2 \\
Pancreaticoduodenectomy & 31 \\
Left hepatic lobectomy + pancreaticoduodenectomy & 1 \\
\hline
\end{tabular}

surgeons who had prior training in the interpretation of pCLE images reviewed the recorded microscopic video sequences and assessed the pCLE images. Pathologists blinded to information from pCLE observation then diagnosed the surgical specimens with standard histopathological techniques. Thereafter, the sensitivity, specificity, positive predictive-value, negative predictive-value, and accuracy of the pCLE findings were determined by comparison with standard histopathological findings. 

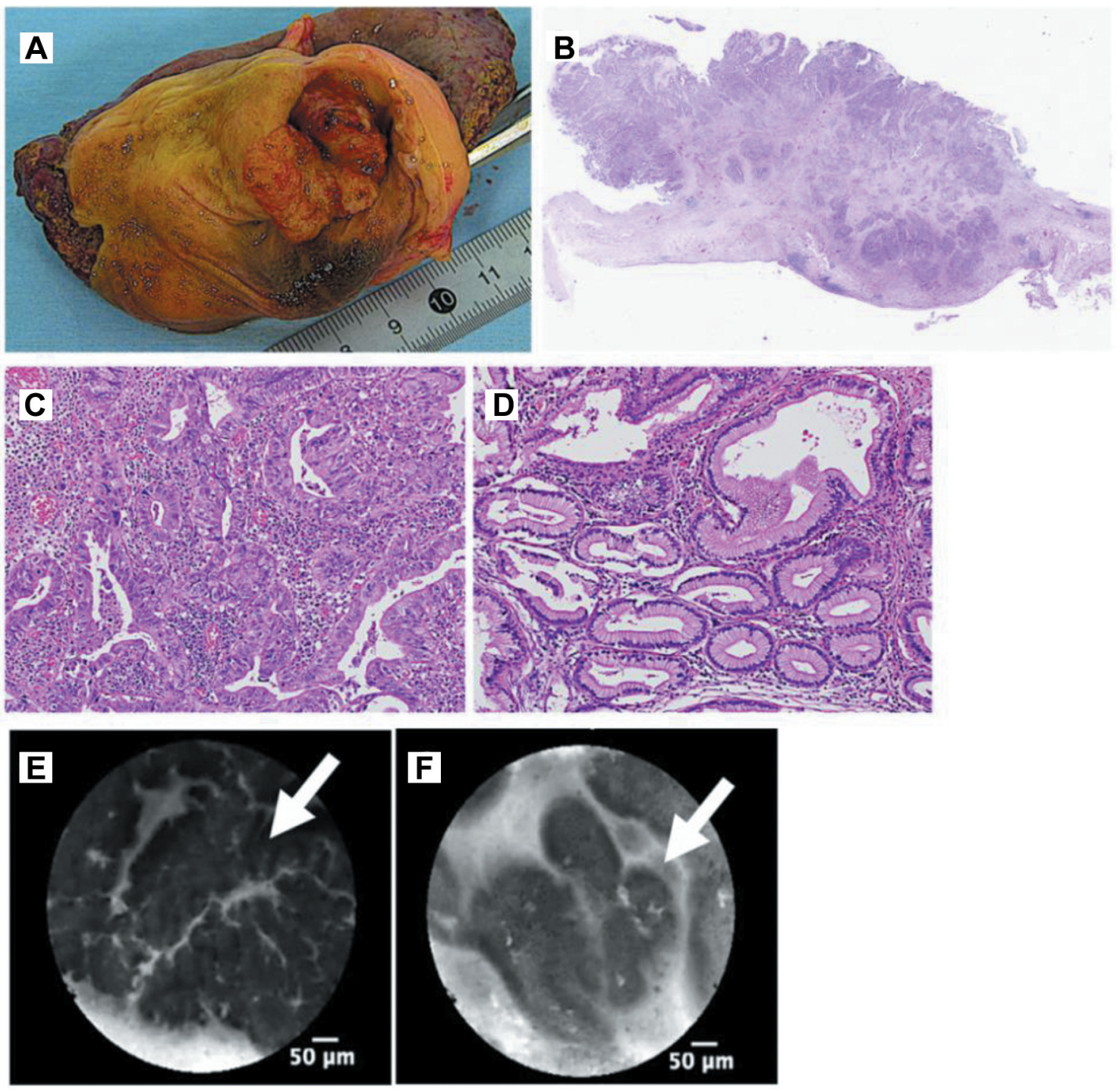

Figure 2. Gallbladder cancer resected from a 72-year-old woman. A: Macroscopic image of the surgical specimen. B: Microscopic image with hematoxylin-eosin (H-E) staining (magnification 40x). C: Microscopic image with H-E staining in the tumor area (magnification 200x). D: Microscopic image with $H$-E staining in the non-tumor area (magnification 200x). E: Probe-based confocal laser endomicroscopy ( $p C L E$ ) image of the tumor area demonstrating the presence of thick dark bands (white arrow), a suspected malignant feature. F: pCLE image of the non-tumor area demonstrating glandular structures without specific malignant features (white arrow).

Statistical analysis. Statistical analyses were conducted using JMP software from SAS (SAS Institute, Cary, NC, USA). The total number of positive findings based on four pCLE-based malignant features of gallbladder lesions was calculated as a continuous function.

\section{Results}

All pathological diagnoses and surgical procedures are summarized in Table I. Video sequences from all patients included were retained for evaluation. Patients with suspected gallbladder malignancy via preoperative diagnosis underwent extended cholecystectomy or bisegmental liver resection.
Surgical gallbladder specimens obtained from other hepatobiliary pancreatic malignancies were also subjected to $e x$ vivo pCLE observation (Table I). The pathology reports showed that no patient who suffered from other hepatobiliary pancreatic malignancies exhibited findings of gallbladder malignancy. Six patients who underwent laparoscopic cholecystectomy for the treatment of cholecystitis were pathologically diagnosed with chronic or gangrenous cholecystitis from pCLE images.

pCLE imaging of gallbladder specimens was performed within 5 min after surgical specimen excision (Figure 2). Suspected malignant gallbladder features based on pCLE 

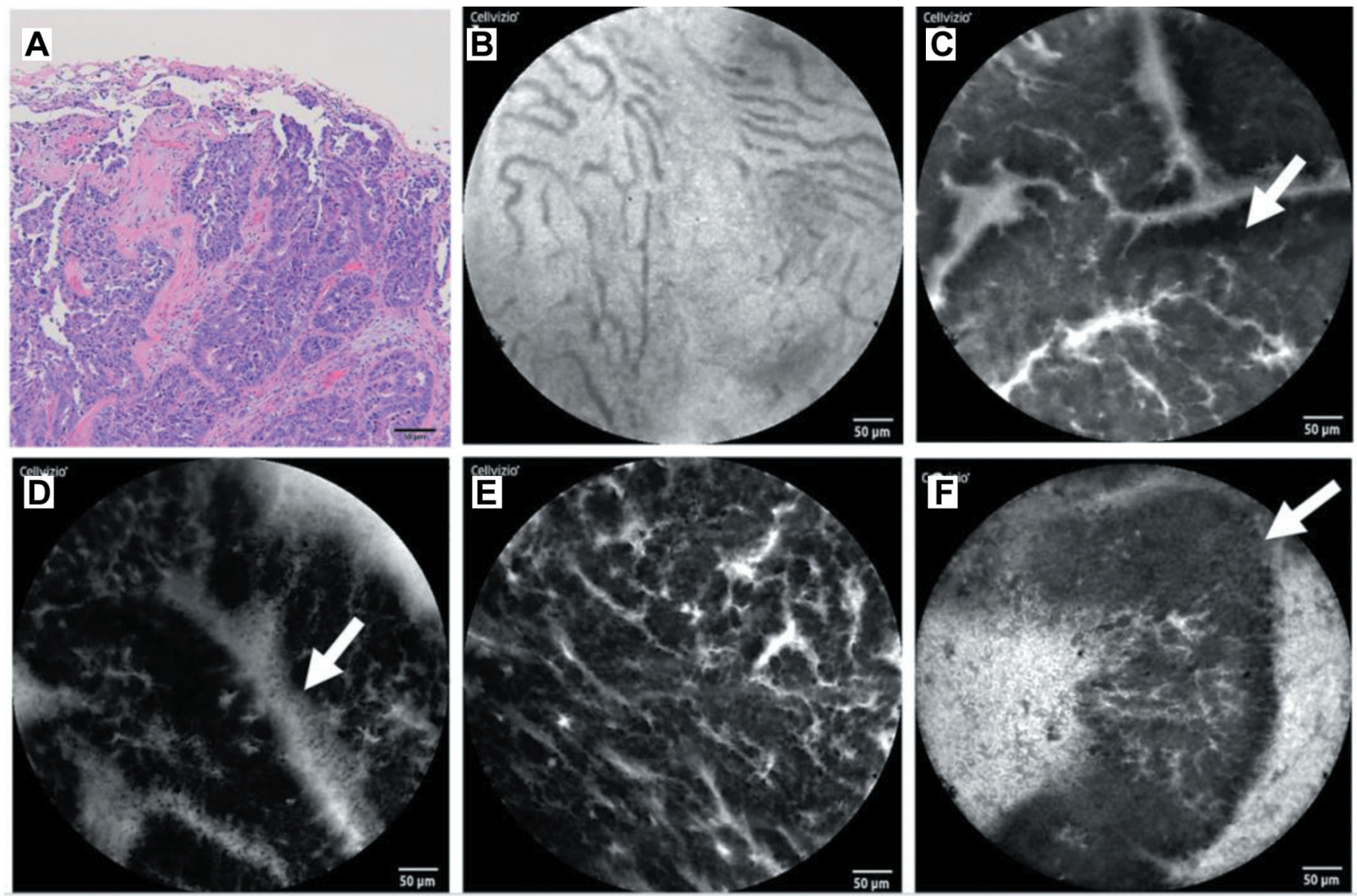

Figure 3. Gallbladder cancer resected from a 63-year-old man. A: Hematoxylin-eosin (H-E) staining in the tumor area (magnification 100x). B: Probe-based confocal laser endomicroscopy ( $p C L E$ ) image of the non-tumor area. $C$ : $p C L E$ imaging revealed thick dark bands in the tumor area (white arrow), a suspected malignant feature. D: pCLE image with thick white bands in the tumor area (white arrow), a suspected malignant feature. $E$ : $p C L E$ image demonstrating dark clumps in the tumor area, a suspected malignant feature. F: $p C L E$ image showing a visualized epithelium in the tumor area (white arrow), a suspected malignant feature.

findings were analyzed according to the Miami Classification, which indicates the following pCLE features to indicate malignancy: thick dark bands $(>40 \mu \mathrm{m})$, thick white bands $(>20 \mu \mathrm{m})$, dark clumps, visualized epithelium, and interstitial fluorescein leakage (8). However, it was not possible to determine interstitial fluorescein leakage since the present study applied fluorescein ex-vivo via spraying. The Paris Classification criteria define the following pCLE findings as benign inflammatory conditions: vascular congestion, roughness aspect, increased interglandular space, and thickened reticular structure (9). The pCLE findings for gallbladder cancer (Figure 3) and cholecystitis (Figure 4) are detailed in Table II and Table III, respectively. None of the patients with cholecystitis whose gallbladder was imaged had thick white bands $(>20 \mu \mathrm{m})$ or visualized epithelium.

Table IV summarizes the sensitivity, specificity, positive predictive-value, negative predictive-value, and accuracy of the Miami Classification for diagnosing gallbladder malignancies. Accordingly, in the present study, when correlated with standard histopathological diagnosis, the presence of thick dark bands $(>40 \mu \mathrm{m})$ and dark clumps had 100\% sensitivity in the diagnosis of gallbladder malignancy, whereas thick white bands $(>20 \mu \mathrm{m})$ and visualized epithelium had $100 \%$ specificity and accuracy.

\section{Discussion}

The accuracy of gallbladder cancer diagnosis was previously unsatisfactory despite the development of pCLE (11-14). The present study demonstrated that pCLE can be utilized for accurate diagnosis of gallbladder malignancy, enabling the differential diagnosis of gallbladder malignancy and benign disease. Several studies have evaluated the role of pCLE for diagnosis of biliary malignancy $(2-6,8,9)$, addressing the challenges associated with the precise characterization of biliary strictures. Accordingly, the Miami Classification provides the criteria for pCLE diagnosis of indeterminate biliary strictures (8), whereas the Paris Classification refines the criteria, improving the specificity and reducing the false- 

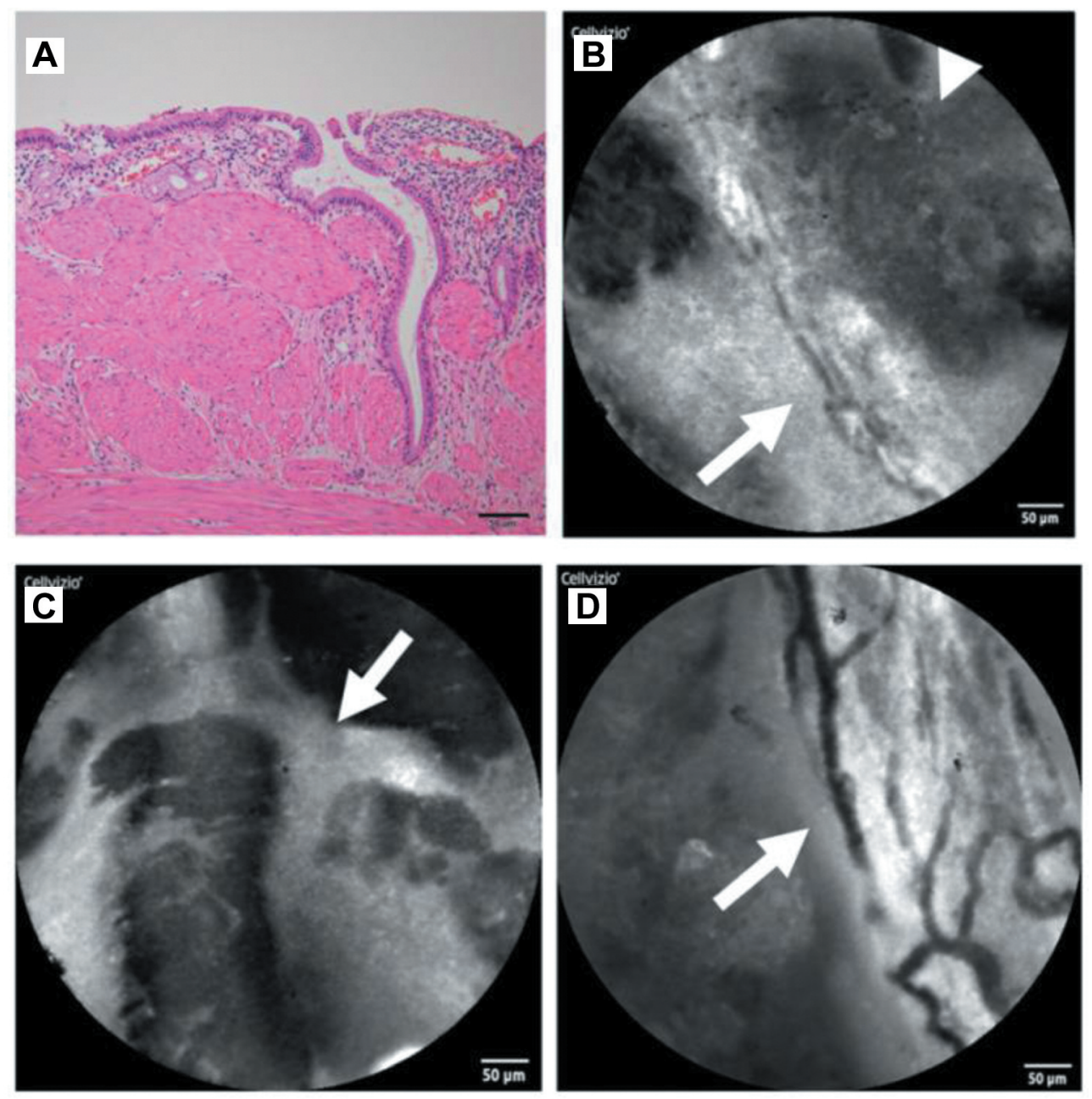

Figure 4. Gallbladder chronic cholecystitis from a 76-year-old man. A: Hematoxylin and eosin staining in the inflammatory area (magnification 100x). B: Probe-based confocal laser endomicroscopy ( $\mathrm{PCLE}$ ) image of the internal surface of the gallbladder with vascular congestion (white arrow) and roughness aspect (white arrow head), a suspected benign inflammatory feature. C: $p C L E$ image demonstrating increased interglandular space (white arrow), a suspected benign inflammatory feature. D: pCLE imaging revealed a thickened reticular structure (white arrow), a suspected benign inflammatory feature.

Table II. Probe-based confocal laser endomicroscopy ( $p C L E)$ findings diagnostic for gallbladder cancer $(n=11)$.

\begin{tabular}{|c|c|c|}
\hline & pCLE finding & $\begin{array}{c}\text { Positive, } \\
\text { n }(\%)\end{array}$ \\
\hline \multirow[t]{4}{*}{ Malignant features } & Thick, dark bands $(>40 \mu \mathrm{m})$ & $11(100)$ \\
\hline & Thick, white bands $(>30 \mu \mathrm{m})$ & $9(81.8)$ \\
\hline & Dark clumps & $11(100)$ \\
\hline & Epithelium visualized & $10(90.9)$ \\
\hline \multirow{4}{*}{$\begin{array}{l}\text { Benign inflammatory } \\
\text { features }\end{array}$} & Vascular congestion & $2(18.2)$ \\
\hline & Roughness aspect & $0(0)$ \\
\hline & Increased interglandular space & $1(9.1)$ \\
\hline & Thickened reticular structure & $0(0)$ \\
\hline
\end{tabular}

Table III. Probe-based confocal laser endomicroscopy ( $p C L E$ ) findings diagnostic for cholecystitis in 62 patients.

\begin{tabular}{llr}
\hline $\mathrm{p}$ & \multicolumn{1}{c}{ CLE finding } & \multicolumn{1}{c}{$\begin{array}{c}\text { Positive, } \\
\mathrm{n}(\%)\end{array}$} \\
\hline Malignant features & Thick, dark bands $(>40 \mu \mathrm{m})$ & $9(14.5)$ \\
& Thick, white bands $(>30 \mu \mathrm{m})$ & $0(0)$ \\
& Dark clumps & $1(17.7)$ \\
& Epithelium visualized & $0(0)$ \\
Benign inflammatory & Vascular congestion & $9(14.5)$ \\
features & Roughness aspect & $12(19.4)$ \\
& Increased interglandular space & $9(14.5)$ \\
& Thickened reticular structure & $34(54.8)$ \\
\hline
\end{tabular}


Table IV. Diagnostic power of probe-based confocal laser endomicroscopy for gallbladder cancer.

\begin{tabular}{|c|c|c|c|c|c|}
\hline Feature & $\begin{array}{c}\text { Sensitivity } \\
(\%)\end{array}$ & $\begin{array}{c}\text { Specificity } \\
(\%)\end{array}$ & $\begin{array}{c}\text { Positive predictive } \\
\text { value }(\%)\end{array}$ & $\begin{array}{c}\text { Negative predictive } \\
\text { value }(\%)\end{array}$ & $\begin{array}{c}\text { Accuracy } \\
(\%)\end{array}$ \\
\hline Thick, dark bands $(>40 \mu \mathrm{m})$ & 100.0 & 85.5 & 55 & 100.0 & 87.7 \\
\hline Thick, white bands $(>30 \mu \mathrm{m})$ & 81.8 & 100.0 & 100 & 96.9 & 97.3 \\
\hline Dark clumps & 100.0 & 82.3 & 50 & 100.0 & 97.3 \\
\hline Epithelium visualized & 90.9 & 100.0 & 100 & 98.4 & 98.6 \\
\hline
\end{tabular}

positive rate (9). To our knowledge, no previous studies have determined gallbladder lesions visualized with pCLE that accurately correlate with histopathologically-determined malignancy.

The present study demonstrated that the presence of thick dark bands ( $>40 \mu \mathrm{m})$ and dark clumps, imaged with pCLE, which have been defined as malignant features in the Miami Classification, correlate with histopathologicallydetermined biliary malignancies. Thick white bands $(>20$ $\mu \mathrm{m})$ and a visualized epithelium, also defined as malignant features in the Miami Classification, were observed in $81.8 \%$ and $90.9 \%$ of malignant cases in the present study, respectively. Our findings also showed that the nonmalignant areas in gallbladder-cancer cases were imaged by pCLE to have a reticular network of thin, dark branching bands and a light gray background, defined as benign features in the Miami Classification. The present results demonstrate that the presence of both thick dark bands $(>40$ $\mu \mathrm{m})$ and dark clumps imaged with pCLE had $100 \%$ sensitivity for malignancy when correlated with standard histopathological criteria. The presence of both thick white bands $(>20 \mu \mathrm{m})$ and visualized epithelium showed $100 \%$ specificity and $97.3 \%$ and $98.6 \%$ accuracy, respectively, for malignancy, when correlated with standard histopathological diagnosis. These findings highlight the potential use of pCLE as an auxiliary diagnostic approach during surgery for gallbladder malignancy.

Vascular congestion and increased interglandular space, which have been defined as features of chronic inflammatory benign disease in the Paris Classification, were observed via pCLE in $18 \%$ and $9 \%$, respectively, of the non-malignant areas of malignant cases in the present study. These results suggest that malignancy is not indicated by chronic inflammatory features visualized with pCLE.

At present, clinicians have the option of inserting a pCLE probe into the gallbladder lumen for preoperative diagnosis of gallbladder cancer. However, the present findings suggest that the use of pCLE to image surgical specimens immediately after resection has the potential to provide accurate information for diagnosing malignant lesions.
Some limitations of the current study are worth noting. Firstly, pCLE examinations were conducted with ex vivo surgical specimens. Moreover, fluorescein was sprayed onto the surface of the gallbladder instead of being intravenously injected, as in previous reports. Therefore, interstitial fluorescein leakage, one of the defined malignant features in the Miami Classification, was not assessed in our cases. However, a previous study reported histopathological similarity of intramucosal fluorescein distribution following either intravenous administration or local mucosal application (10). It should be noted that pCLE findings analogous to malignant features in the Miami Classification were identified in the present study in a small number of cases that were histopathologically diagnosed as cholecystitis. These results suggest that further investigation and discovery of other pathognomonic findings are required to fully establish the criteria for diagnosing gallbladder cancer using pCLE. Other limitations include the small sample size, single-center design, and unavailability of frozen sections for diagnosis with pCLE. Further large-scale, multi-center studies would certainly confirm the initial findings demonstrated in this study.

In conclusion, the present study has demonstrated pCLE has the potential to improve current limitations for accurately diagnosing gallbladder malignancy.

\section{Conflicts of Interest}

There are no conflicts of interest.

\section{Authors' Contributions}

TK, TA and YT designed and performed experiments, analyzed data and wrote the article; TY, DAM, TY, TK, KM, YW, HS, KS, KN, $\mathrm{T}$ H, KT, TH, AF, AEAG, and RMH gave technical support and conceptual advice. RMH revised the manuscript.

\section{Acknowledgements}

In memoriam: Tomotake Koizumi, MD, Ph.D.

Tragically and sadly Dr. Koizumi passed away shortly after submission of the present paper at the young age of 47 . Dr. Koizumi was a pioneer and world leader in fluorescence-based 
tumor analysis and fluorescence-guided cancer surgery. Dr. Koizumi was a beloved colleague, friend and teacher, and his sudden and unexpected passing has devastated all who knew him. The present study, Dr. Koizumi's last paper, will serve as a lasting memorial of him.

\section{References}

1 Rawla P, Sunkara T, Thandra KC and Barsouk A: Epidemiology of gallbladder cancer. Clin Exp Hepatol 5(2): 93-102, 2019. PMID: 31501784. DOI: 10.5114/ceh.2019.85166

2 Wallace MB and Fockens P: Probe-based confocal laser endomicroscopy. Gastroenterology 136(5): 1509-1513, 2009. PMID: 19328799. DOI: 10.1053/j.gastro.2009.03.034

3 Meining A, Chen YK, Pleskow D, Stevens P, Shah RJ, Chuttani $\mathrm{R}$, Michalek $\mathrm{J}$ and Slivka A: Direct visualization of indeterminate pancreaticobiliary strictures with probe-based confocal laser endomicroscopy: a multicenter experience. Gastrointest Endosc 74(5): 961-968, 2011. PMID: 21802675. DOI: $10.1016 /$ j.gie.2011.05.009

4 Slivka A, Gan I, Jamidar P, Costamagna G, Cesaro P, Giovannini M, Caillol $\mathrm{F}$ and Kahaleh M: Validation of the diagnostic accuracy of probe-based confocal laser endomicroscopy for the characterization of indeterminate biliary strictures: results of a prospective multicenter international study. Gastrointest Endosc 81(2): 282-290, 2015. PMID: 25616752. DOI: 10.1016/j.gie.2014.10.009

5 Yang JF, Sharaiha RZ, Francis G, Karia K, Kistler CA, Laique SN, Hasan R, Gaidhane M, Tyberg A, Kahaleh M, Kowalski TE, Loren DE and Siddiqui A: Diagnostic accuracy of directed cholangioscopic biopsies and confocal laser endomicroscopy in cytology-negative indeterminate bile duct stricture: a multicenter comparison trial. Minerva Gastroenterol Dietol 62(3): 227-233, 2016. PMID: 27304199.

6 Taunk P, Singh S, Lichtenstein D, Joshi V, Gold J and Sharma A: Improved classification of indeterminate biliary strictures by probe-based confocal laser endomicroscopy using the Paris Criteria following biliary stenting. J Gastroenterol Hepatol 32(10): 1778-1783, 2017. PMID: 28294404. DOI: 10.1111/jgh.13782

7 Konda VJ, Meining A, Jamil LH, Giovannini M, Hwang JH, Wallace MB, Chang KJ, Siddiqui UD, Hart J, Lo SK, Saunders MD, Aslanian HR, Wroblewski K and Waxman I: A pilot study of in vivo identification of pancreatic cystic neoplasms with needle-based confocal laser endomicroscopy under endosonographic guidance. Endoscopy 45(12): 1006-1013, 2013. PMID: 24163192. DOI: 10.1055/s-0033-1344714
8 Meining A, Shah RJ, Slivka A, Pleskow D, Chuttani R, Stevens $\mathrm{PD}$, Becker V and Chen YK: Classification of probe-based confocal laser endomicroscopy findings in pancreaticobiliary strictures. Endoscopy 44(3): 251-257, 2012. PMID: 22261749. DOI: $10.1055 / \mathrm{s}-0031-1291545$

9 Caillol F, Filoche B, Gaidhane M and Kahaleh M: Refined probe-based confocal laser endomicroscopy classification for biliary strictures: the Paris Classification. Dig Dis Sci 58(6): 1784-1789, 2013. PMID: 23314855. DOI: 10.1007/s10620-0122533-5

10 Nonaka K, Ohata K and Nakai Y: Probe-based confocal laser endomicroscopy of the duodenal mucosa with fluorescein dispersion. Dig Endosc 26(4): 604, 2014. PMID: 24702265. DOI: $10.1111 /$ den.12296

11 Rodríguez-Fernández A, Gómez-Río M, Medina-Benítez A, Moral JV, Ramos-Font C, Ramia-Angel JM, Llamas-Elvira JM, FerrónOrihuela JA and Lardelli-Claret P: Application of modern imaging methods in diagnosis of gallbladder cancer. J Surg Oncol 93(8): 650-664, 2006. PMID: 16724342. DOI: 10.1002/jso.20533

12 Zemour J, Marty M, Lapuyade B, Collet D and Chiche L: Gallbladder tumor and pseudotumor: Diagnosis and management. J Visc Surg 151(4): 289-300, 2014. PMID: 24930718. DOI: $10.1016 /$ j.jviscsurg.2014.05.003

13 Jang JY, Kim SW, Lee SE, Hwang DW, Kim EJ, Lee JY, Kim SJ, Ryu JK and Kim YT: Differential diagnostic and staging accuracies of high resolution ultrasonography, endoscopic ultrasonography, and multidetector computed tomography for gallbladder polypoid lesions and gallbladder cancer. Ann Surg 250(6): 943-949, 2009. PMID: 19855259. DOI: 10.1097/SLA.0b013e3181b5d5fc

14 Bo X, Chen E, Wang J, Nan L, Xin Y, Wang C, Lu Q, Rao S, Pang L, Li M, Lu P, Zhang D, Liu H and Wang Y: Diagnostic accuracy of imaging modalities in differentiating xanthogranulomatous cholecystitis from gallbladder cancer. Ann Transl Med 7(22): 627, 2019. PMID: 31930028. DOI: 10.21037/atm.2019.11.35

Received October 17, 2021

Revised November 13, 2021

Accepted November 30, 2021 